\title{
Acoustic and seismic modalities for unattended ground sensors
}

\author{
Gerard E. Sleefe, Mark D. Ladd, Timothy S. McDonald, and Gregory J. Elbring \\ Sandia National Laboratories, MS 0859, Albuquerque, NM 87185-0859
}

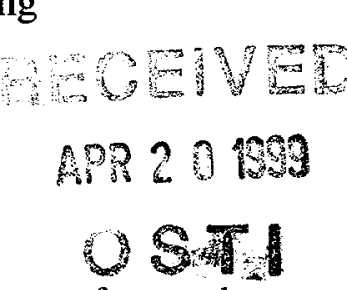

\begin{abstract}
The use of acoustic and seismic modalities in unattended ground sensor systems has been an active area of research. However, a thorough study of the advantages and complementary aspects of the two modalities has not heretofore been considered. This paper provides a detailed description of both acoustic and seismic methods for unattended ground sensors. We discuss acoustic and seismic phenomenology; source mechanisms, propagation paths, attenuation, and sensing. These phenomena significantly impact the detection, identification, and localization objectives of unattended ground sensors. As such, certain applications will benefit from exploiting acoustic ground sensing, seismic ground sensing, or in some cases, both. A methodology is presented for selecting the preferred modality (acoustic and/or seismic) for a particular application. It is shown that the preferred modality significantly impacts the architecture of the ground sensor system. Important system architecture criteria such as transducer selection, data acquisition bandwidth, array geometry, and communications requirements are considered in this paper. Furthermore, the effects of seismo-acoustic and acousto-seismic interactions are addressed. It is shown that acoustic transducers often respond to seismic excitation, and seismic transducers often respond to acoustic excitation. In some instances, this dual-modality is of benefit. We also provide recommendations for minimizing seismic/acoustic cross talk for applications requiring independent seismic or acoustic modes.
\end{abstract}

Keywords: acoustic, seismic, sensor, system, autonomous

\section{INTRODUCTION}

Unattended ground sensors (UGS) are used for remote sensing in a variety of applications ranging from industrial monitoring to military information gathering. As the name implies, an UGS is a device which is placed on the ground, automatically gathers sensor data on a remote "target", interprets the data, and communicates information back to some other location. UGS are capable of gathering and interpreting data on the remote target without interaction from a human operator. UGS are set at some standoff distance from the target and monitor emanations from the target. Both acoustic and seismic emanations have been given considerable attention in practical unattended ground sensor systems. The particular sensing modality (acoustic or seismic) depends on the application and the target(s) of interest. For example, ground sensor systems for detecting overhead aircraft are generally based on acoustic principles since the source (engine noise), and coupling medium (air) are primarily acoustic in nature. On the other hand, the detection of underground mining activities, such as deep blasting, would require a seismic UGS. There are certain applications where both acoustic and seismic emanations coexist. An example of this coexistence is detection of moving vehicles: acoustic energy emanates from the engine and exhaust, and seismic energy emanates as the rolling tires contact the roadbed. Further complicating many applications is a coupling between the acoustic and seismic modes. In the moving vehicle example, some of the acoustic energy emanating from the exhaust strikes the ground and converts into seismic energy. It is therefore essential that ground sensors properly exploit acoustic and/or seismic modalities to detect, identify, and locate targets.

The primary objective of this paper is to study the relative advantages and complementary aspects of acoustic and seismic ground sensors. In the next section, a detailed description of both acoustic and seismic ground sensing methods is provided. Acoustic and seismic phenomenology including source mechanisms, propagation paths, attenuation, and sensing is discussed in detail. Also described in Section 2 are the effects of seismo-acoustic and acousto-seismic interactions as well as recommendations for minimizing seismic/acoustic cross talk. In Section 3, representative acoustic and seismic ground sensor data are presented. The data illustrate that acoustic transducers may respond to seismic excitation, and seismic transducers often respond to acoustic excitation. In Section 4, we describe the implications of the phenomenology on the detection, identification, and localization objectives of unattended ground sensors. A methodology is presented for selecting the preferred modality (acoustic and/or seismic) for a particular application. The implications of the selected modality on the system architecture are also discussed. This paper concludes with a summary of the advantages and complementary aspects of acoustic and seismic ground sensors. 


\section{DISCLAIMER}

Portions of this document may be illegible in electronic image products. Images are produced from the best available original document. 


\section{ACOUSTIC AND SEISMIC MODALITIES}

\subsection{Acoustic ground sensing and phenomenology}

The concept of acoustic ground sensing is illustrated in Figure 1a. An acoustic ground sensor system is used to detect, identify, and locate sources of acoustic emanations. The acoustic emanations result from dynamic pressure differentials occurring at the target. Sources of such pressure differentials include exhausted air and vibrating components [1]. The acoustic emanations from the target propagate as compression waves with a nominal sound speed of $330 \mathrm{~m} / \mathrm{sec}$, and potentially impinge upon the acoustic transducer at the ground sensor system. The acoustic transducer, commonly referred to as a microphone, produces a voltage that is proportional to the incident dynamic acoustic pressure. Omni-directional microphones based on condenser or piezoelectric technologies are generally the appropriate transducers for acoustic ground sensors. These transducers provide excellent sensitivity over a frequency range of $20 \mathrm{~Hz}$ to $10,000 \mathrm{~Hz}$. When coupled with low-noise pre-amplifiers, microphones can provide a dynamic range in excess of $120 \mathrm{~dB}$. In practice, however, the noisefloor of microphones is dominated by background acoustic sources, especially wind noise. For example, a 10-km/hr wind will result in a 30-dB increase in the background noise relative to quiescent conditions [2]. Wind noise therefore can significantly reduce the performance of an acoustic ground sensor system.

The phenomenology of acoustic ground sensing is significantly influenced by both atmospheric and terrain variations. In the ideal case of an isotropic atmosphere and flat terrain, an acoustic wave will follow a straight path between the target and the sensor. In the more general case, however, as illustrated in Figure 1b, atmospheric and terrain effects result in reflection, refraction, scattering, and attenuation. This phenomenology significantly affects the acoustic signal level at the ground sensor. For example, it is quite possible that the atmosphere and/or terrain can form a 'shadow zone', such that a ground sensor placed in this zone would be unable to detect emanations from the target. Furthermore, because atmospheric conditions change with time, the acoustic signal level at the ground sensor can exhibit both diurnal and seasonal variations.

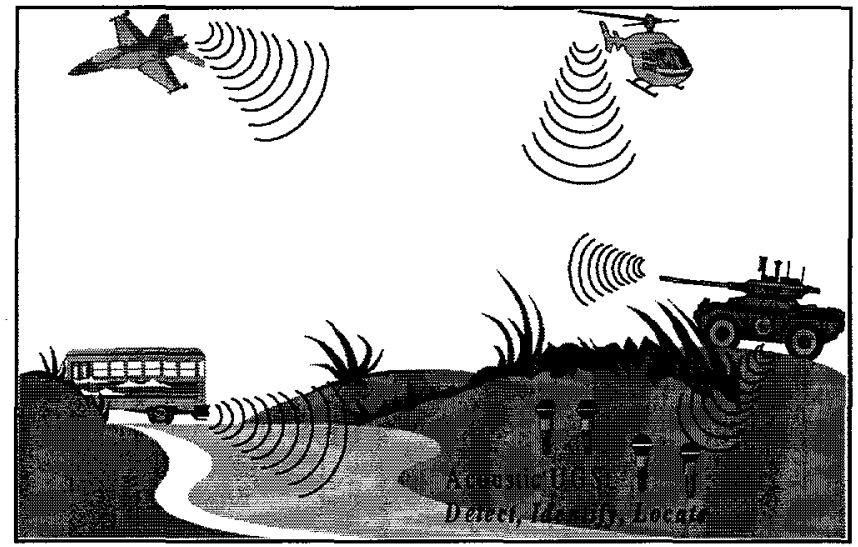

(a)

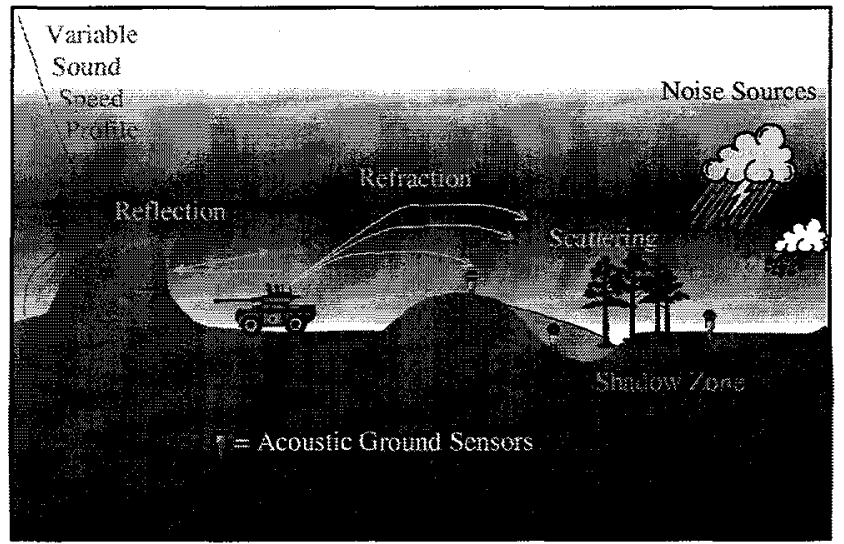

(b)

Figure 1. a.) Acoustic unattended ground sensor concept and b.) Acoustic phenomenology

Another practical consideration of acoustic ground sensors is frequency-dependent and range-dependent propagation effects. Acoustic absorption, scattering, and spherical spreading result in a decrease in signal amplitude at both increasing ranges and increasing frequencies. Hence, the ability of a ground sensor system to detect and identify a target is a function of its emanation frequencies, and the range between target and sensor. As a result, acoustic ground sensors nominally perform best when operating in the frequency band of $20-1000 \mathrm{~Hz}$ at target-sensor-ranges of $100-600 \mathrm{~m}$ [3].

A final important consideration is the variability of the sound speed in air. For example, the temperature-dependence of the sound speed in air can be approximately represented by:

$$
c \cong c_{0} \sqrt{1+T / 273}
$$


where $\mathrm{c}_{0}$ is the sound speed at $0^{\circ} \mathrm{C}(331.6 \mathrm{~m} / \mathrm{s})$, and $\mathrm{T}$ is the temperature in degrees Celsius. So at $30^{\circ} \mathrm{C}$, the sound speed is approximately $5 \%$ faster than at $0^{\circ} \mathrm{C}$. Such a small sound speed variation alone would result in a relatively small uncertainty when estimating target locations by the time-of-flight method. However, additional atmospheric effects such as temperature inversions, can cause significant refraction and reflection phenomena to occur, as illustrated in Figure $1 \mathrm{~b}$. Such refraction and reflection phenomena, if not properly accounted for, introduce a significant error in target location estimates.

\subsection{Seismic ground sensing and phenomenology}

The concept of seismic ground sensing is illustrated in Figure 2a. A seismic ground sensor system is used to detect, identify, and locate sources of seismic emanations. Seismic emanations occur when vibrating components within the target are mechanically coupled to the ground. Target vibrations that are coupled into the earth propagate within the ground as elastic waves. These elastic waves propagate in a complex manner through the geologic medium, and potentially impinge upon the seismic transducer at the ground sensor system. The seismic transducer measures the earth's vibrations at a single point. Two types of seismic transducers are commonly used within ground sensor systems. The first type, commonly referred to as a geophone, is a moving-coil electro-dynamic device that produces a voltage proportional to the velocity of the ground vibrations. Geophones exhibit both a low-frequency fundamental resonance and a high-frequency spurious resonance. The manufacturer meticulously specifies the resonant frequency of commercial geophones, with common resonant frequencies being $10 \mathrm{~Hz}, 14 \mathrm{~Hz}, 28 \mathrm{~Hz}$, and $40 \mathrm{~Hz}$. The spurious resonance nominally occurs at 25 -times the fundamental resonance, and is often specified by the manufacturer. Because of amplitude and phase distortions at the fundamental and spurious resonant frequencies, ground sensor systems should generally exploit only those frequencies between the fundamental and spurious resonances. For example, when using a $14 \mathrm{~Hz}$ geophone, the recommended seismic bandwidth is from $15 \mathrm{~Hz}$ to $340 \mathrm{~Hz}$. For wider bandwidth systems, accelerometers are often considered. Accelerometers are solid-state piezo-electric devices that produce a voltage proportional to the acceleration of the ground vibrations. Accelerometers exhibit a high-frequency resonant frequency and no low-frequency resonances. Typical seismic accelerometers have a resonant frequency in excess of $1000 \mathrm{~Hz}$. Hence, accelerometers can be used over a bandwidth of $0-\mathrm{Hz}$ to $1000 \mathrm{~Hz}$. Unfortunately, current seismic accelerometers are fragile and exhibit excessive electronic noise at low frequencies, thereby limiting their usefulness. It has been shown that geophones are preferred for sensing frequencies below approximately $100 \mathrm{~Hz}$, while accelerometers are preferred for sensing frequencies above $100 \mathrm{~Hz}$ [4]. In many ground sensor applications, the majority of propagated seismic energy is below $100 \mathrm{~Hz}$, and thus geophones suffice.

Both wind noise and cultural noise influence the performance of seismic ground sensors. Wind noise may couple into seismic ground sensors by either direct or indirect means. Direct wind noise coupling results when some portion of the ground sensor is in direct contact with the wind. For example, if the ground sensor is a rigid body with an antenna that extends into the air, air motion across the antenna can couple into the transducer. Completely burying the sensor or mechanically decoupling exposed elements from the transducer can minimize direct wind noise. Indirect wind noise coupling occurs when the wind strikes the ground and/or vegetation in the vicinity of the ground sensor. Wind-induced vibrations result from the reaction of the ground to the wind force and through root motions. As shown in a subsequent section, burying the transducer at significant depths can reduce indirect wind noise.

Seismic wave propagation is highly dependent on the underlying geology. As illustrated in Figure $2 b$, seismic propagation can include compression waves, shear waves, surface (Rayleigh) waves, reflected waves, and refracted waves. Whereas acoustic waves propagate at a near-constant velocity, seismic wave speeds can range from $200 \mathrm{~m} / \mathrm{sec}$ (surface waves in loose, dry sediments) to over $4000 \mathrm{~m} / \mathrm{s}$ (compression waves in hard rock). As a result, localization of targets using seismic ground sensors is a challenging problem. Fortunately, geology is not time-dependent, so advanced localization methods such as travel-time-inversion and F-K beam-forming can be effective when combined with seismic propagation models and/or active seismic calibrations.

Frequency-dependent and range-dependent propagation effects also influence seismic ground sensors [5]. Seismic absorption, scattering, and spherical spreading result in a decrease in signal amplitude at both increasing ranges and increasing frequencies. Because these effects are highly dependent on the geologic medium, the operating bandwidth and required target-to-sensor range is highly variable. In practice, seismic ground sensor systems are designed for a nominal bandwidth (e.g. $20 \mathrm{~Hz}-400 \mathrm{~Hz}$ ), and then deployed at a target-to-sensor range appropriate for the presumed geology and target strengths. 


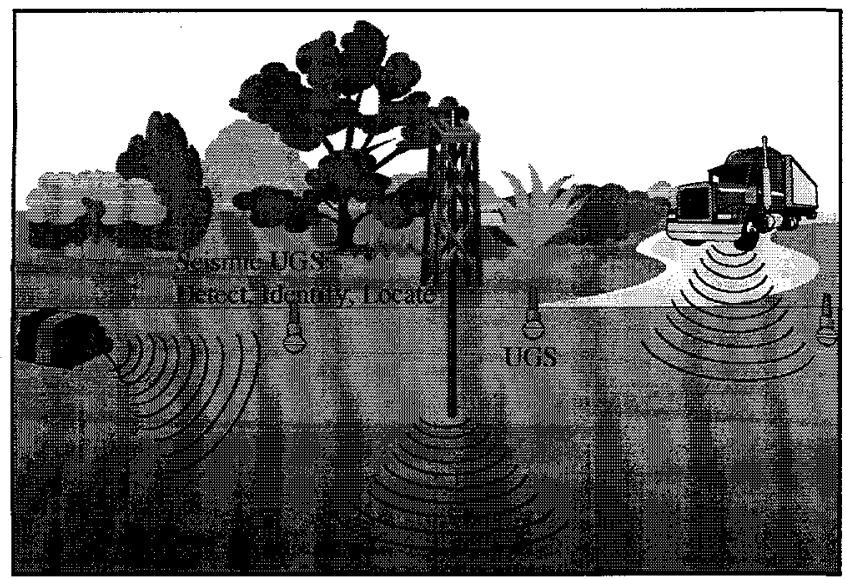

(a)

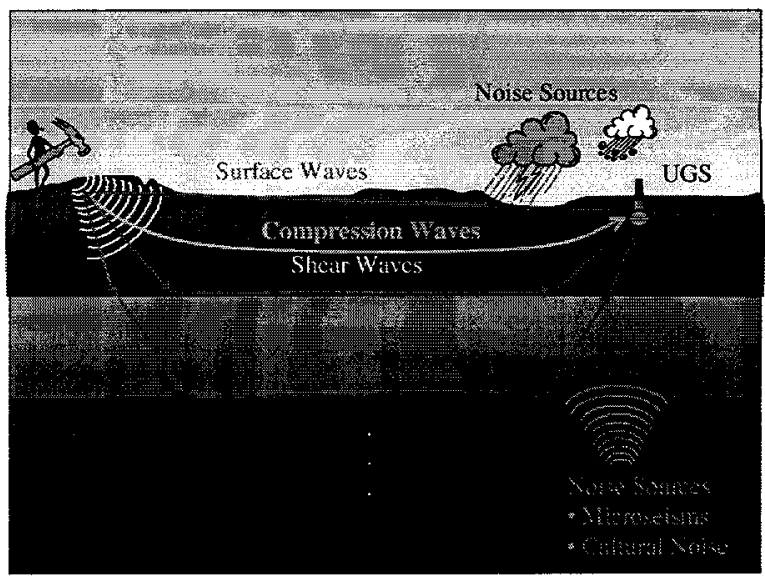

(b)

Figure 2. a) Seismic ground sensing concept and b) Seismic phenomenology

\subsection{Seismo-acoustic and acousto-seismic interactions}

Acoustic and seismic phenomenologies were independently addressed in Sections 2.1 and 2.2 respectively. Under certain circumstances, however, seismo-acoustic and acousto-seismic interactions need to be considered. An acousto-seismic interaction is defined as an airborne acoustic wave that couples into the ground and/or the seismic transducer. When an airborne acoustic wave impinges on the earth's surface, some portion of the energy is converted to seismic energy and propagates into the ground. These seismo-acoustic waves generally propagate from source-to-sensor at the acoustic velocity $(331 \mathrm{~m} / \mathrm{sec})$ and can be measured by a surface geophone. The acousto-seismic wave is rapidly attenuated as it propagates into the earth, and is usually observed only in seismic sensors at or near the surface. The acousto-seismic interaction can be amplified if some portion of the seismic ground sensor system extends into the air, thereby creating a reflecting surface reaction.

A seismo-acoustic interaction is defined as a ground-borne seismic wave that couples into the air and/or the acoustic transducer. In general, seismo-acoustic mode conversion at the surface is very inefficient and usually not observed in airborne acoustic sensors. However, microphone elements that are mechanically coupled to the ground can produce a signal in response to surface seismic motions. Placing the microphone in a vibration isolation mount can minimize this seismoacoustic interaction.

\section{EXPERIMENTAL METHODS AND RESULTS}

\subsection{Sensor noise experiment}

An experiment was undertaken to illustrate wind-induced seismic noise. For this experiment, a remote location in Nevada was selected so as to minimize cultural seismic noise sources. At the site, three $10 \mathrm{~Hz}$ geophones were buried at depths of $0.5 \mathrm{~m}, 1.0 \mathrm{~m}$, and $2.0 \mathrm{~m}$ respectively. Data was collected simultaneously on all three geophones under $25 \mathrm{~km} / \mathrm{hr}$ nominal wind conditions. At these wind speeds, the wind-induced noise dominated over the low seismic cultural noise background. Raw time series data was collected for a period of 1 minute and the periodogram method was applied to produce a noise power spectral density measurement. The power spectrum from the $0.5 \mathrm{~m}$ sensor was used to normalize the deeper sensor data and estimate the signal attenuation as a function of depth. The resulting attenuation of wind-induced noise measurements over the frequency range of $10-70 \mathrm{~Hz}$ is plotted in Figure 3. Note that the wind-induced noise decreases as the geophone depth-ofburial increases. Furthermore note that high frequency wind noise is more rapidly attenuated than lower frequency noise. These depth and frequency dependencies are manifestations of the acousto-seismic nature of wind-noise. In particular, windnoise within seismic ground sensors can be considerably reduced by appropriately burying the sensor. Reduction of windnoise in this manner can significantly increase the detection performance of seismic unattended ground sensor systems. 


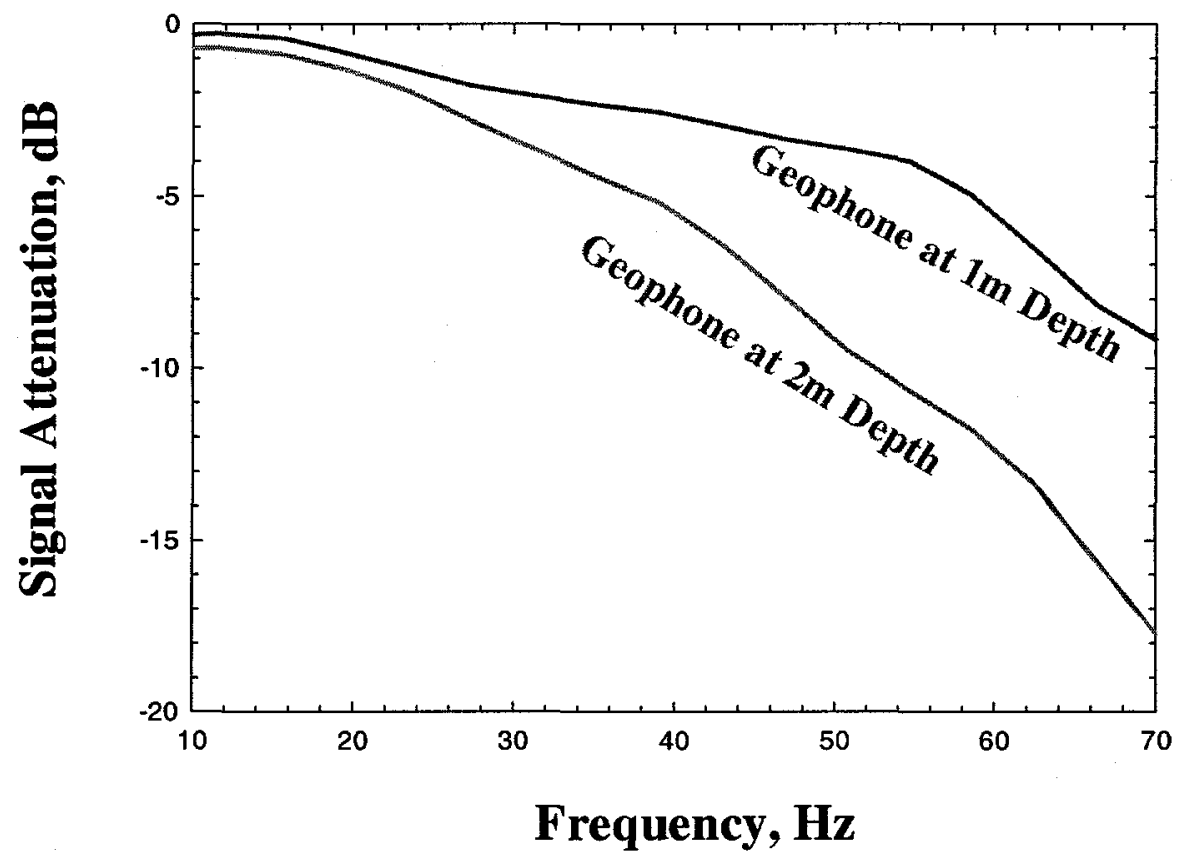

Figure 3. Attenuation of wind-induced seismic noise, referenced to a geophone at $0.5 \mathrm{~m}$ depth, for depths-of-burial of $1.0 \mathrm{~m}$ and $2.0 \mathrm{~m}$. Note that wind-induced seismic noise decreases as a function of both depth and frequency.

\subsection{Surface detonation experiment}

An experiment was undertaken to demonstrate coupling between acoustic and seismic propagation modes. In this experiment, a $150 \mathrm{lb}$ explosive charge was detonated at the surface (local geology is dry alluvium), thereby simultaneously generating an airborne acoustic pulse and a well-coupled seismic wave. A $28-\mathrm{Hz}$ geophone was deployed at a range of $800 \mathrm{~m}$ from the detonation. The geophone was buried $0.5 \mathrm{~m}$ below the surface to avoid any direct acoustic coupling. A broadband microphone was also deployed at this $800 \mathrm{~m}$ range. The microphone was placed in a vibration isolation stand at a height of $0.5 \mathrm{~m}$ above the ground, thereby minimizing any direct seismic coupling. Figure 4a plots the simultaneous time series recorded by the geophone and the microphone. Note that the microphone data primarily consists of an air-borne detonation pulse. The first arrival on the microphone data occurs at 2.39 seconds after the detonation. For the $800 \mathrm{~m}$ source-receiver separation, this corresponds to a propagation speed of $333 \mathrm{~m} / \mathrm{s}$, thus verifying the pure acoustic path of the microphone signal.

Referring to the seismic trace in Figure 4a, significant seismic propagation complexity was observed. An initial broadband pulse arrives at the geophone at 0.6 seconds after the detonation. This corresponds to a propagation velocity of $1333 \mathrm{~m} / \mathrm{s}$, consistent with compressional seismic wave propagation in dry alluvium. Also note that the seismic sensor clearly records the acoustic arrival. This directly confirms the presence of an acousto-seismic interaction. Figure $4 \mathrm{~b}$ shows an enlarged portion of the acoustic arrivals. An acoustic reverberation from the detonation is observed in both the geophone and microphone data. Furthermore, note that the only the geophone signal contains a low frequency wavelet prior to the acoustic arrival. This wavelet travels slightly faster than the acoustic velocity and is the result of a surface seismic wave.

\subsection{Idled vehicle experiment}

To further illustrate acoustic and seismic ground sensor modalities, an idled vehicle experiment was undertaken. In this experiment, a large diesel truck was idled at constant speed on a dirt road. In an idled mode, the truck emanates primarily acoustic energy through its exhaust. Both a geophone (buried $0.5 \mathrm{~m}$ ) and a microphone (vibration isolated at $0.5 \mathrm{~m}$ above surface) were deployed at a range of $200 \mathrm{~m}$ from the vehicle. A simultaneous 1-minute time series was acquired from the two sensors. The periodiogram method was applied, and the resulting geophone and microphone spectra are provided in Figure 5. Note that many of the truck emanation tones appear in both the geophone and microphone data. However, at high frequencies (above $100 \mathrm{~Hz}$ ), the microphone data clearly shows tones not observed in the geophone data. The high frequency attenuation of the geophone information may imply that the tones are propagated by acoustic paths and coupled to the 
geophone through an acousto-seismic interaction. On the other hand; the geophone exhibits several low-frequency tones (e.g. 20 and $30 \mathrm{~Hz}$ ) that do not appear in the microphone spectra. These low-frequency tones suggest that some emanations from the truck couple directly to the ground (e.g. through the wheels) and propagate seismically to the ground sensor. Further study, such as velocity analysis with frequency-wavenumber methods [6] is required to ascertain the propagation paths of the various tones. However, the fact that the geophone and microphone provide independent spectral information suggests that the fusion of seismic and acoustic modalities may provide improved methods for detecting and identifying vehicles.
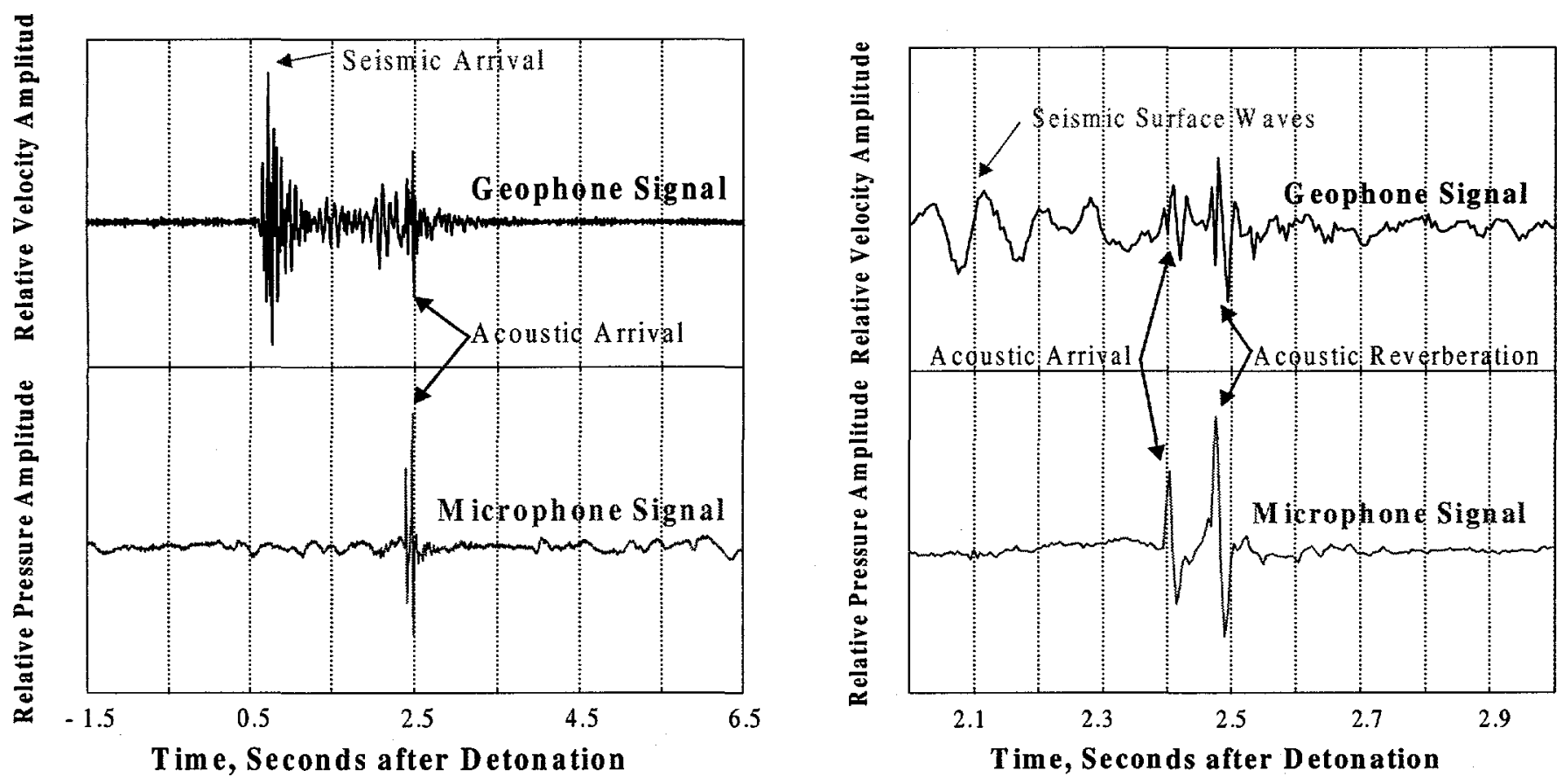

Figure 4. a.) Geophone and microphone simultaneous recording of an explosive detonation at $800 \mathrm{~m}$ range, and b.) enlarged portion of a) around the time of the acoustic arrivals.

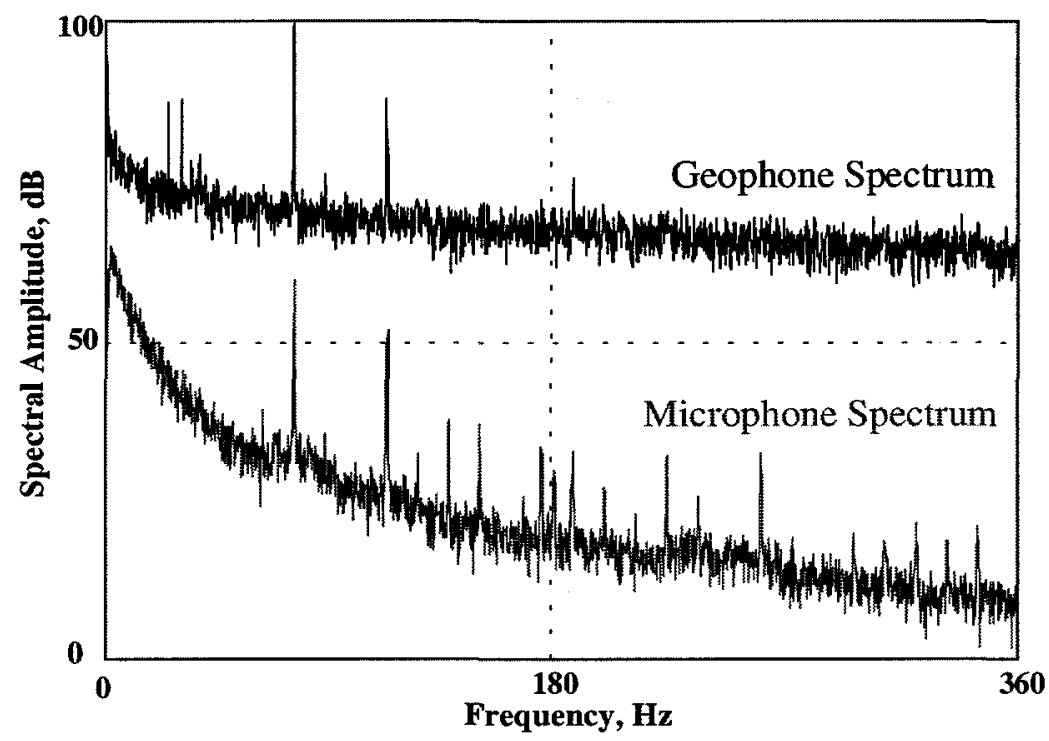

Figure 5. Geophone and microphone simultaneous recording of a large diesel truck at $200 \mathrm{~m}$ range 


\section{DISCUSSION}

\subsection{Implications on target detection, identification, and localization}

As illustrated in the previous sections, both acoustic and seismic modalities can provide important information on target emanations. The selection of the appropriate methodology is dependent on a number of factors, as described in the next section. However, it is important to outline first the implications that acoustic and seismic phenomenologies have on the detection, identification, and localization performance of ground sensors. Table 1 provides a matrix of these implications for seismic and acoustic modalities.

Table 1: Target Detection, Identification, and Localization Issues attributed with acoustic and seismic UGS

\begin{tabular}{|l|l|l|}
\hline Issue & Acoustic Modality & Seismic Modality \\
\hline $\begin{array}{l}\text { Target Detection } \\
\text { Detection decreases with range }\end{array}$ & Yes, moderate & Yes, significant in poor geology \\
Potential for 'shadow zones' & Yes, significant & Yes, if geology complex \\
Diurnal/seasonal effects & Yes, significant & Yes, minimal \\
Can detect air-borne emanations & Yes & Yes, via acousto-seismic interact \\
Can detect subsurface emanations & Not likely & Yes \\
Target Identification \\
High-Frequency Harmonics \\
$\begin{array}{l}\text { Low-Frequency Vibrations } \\
\text { Target Localization } \\
\text { Time-of-flight method }\end{array}$ & Yes & No \\
Array Bearing Method & No & Yes \\
\end{tabular}

\subsection{Methodology for selecting appropriate modality}

Having established the advantages and disadvantages of both acoustic and seismic ground sensors, we are now in a position to establish a methodology for selecting the appropriate modality. The modality that is selected will primarily depend on two factors: the source of the acoustic/seismic emanations, and the intended ground sensor mission. For example, airborne sources, such as aircraft, are best monitored with acoustic UGS. On the other hand, subsurface sources such as underground mining are best monitored with seismic UGS. In many applications, both seismic and acoustic emanations coexist, and the fusion of acoustic and seismic ground sensor data may be synergistic. Hence, the methodology for selecting the appropriate ground sensor modality is summarized in Figure 6.

\subsection{Implications of selected modality on system architecture}

Having identified the preferred modality (acoustic, seismic, or a combination), certain system architecture aspects need be considered. These considerations relate to deployment means, data acquisition and telemetry requirements. For example, since seismic UGS perform best when buried, they can be effectively deployed by airdrop, but would require excavation for hand-delivery. On the other hand, acoustic UGS require a microphone appendage in the air and are thus more difficult to 
deploy by airdrop. Also, acoustic UGS generally require larger data acquisition bandwidths, thereby impacting the data acquisition, signal processing, data storage, and telemetry requirements. These issues, along with mission specific concept of operations must be considered when selecting acoustic, seismic, or mixed modalities.

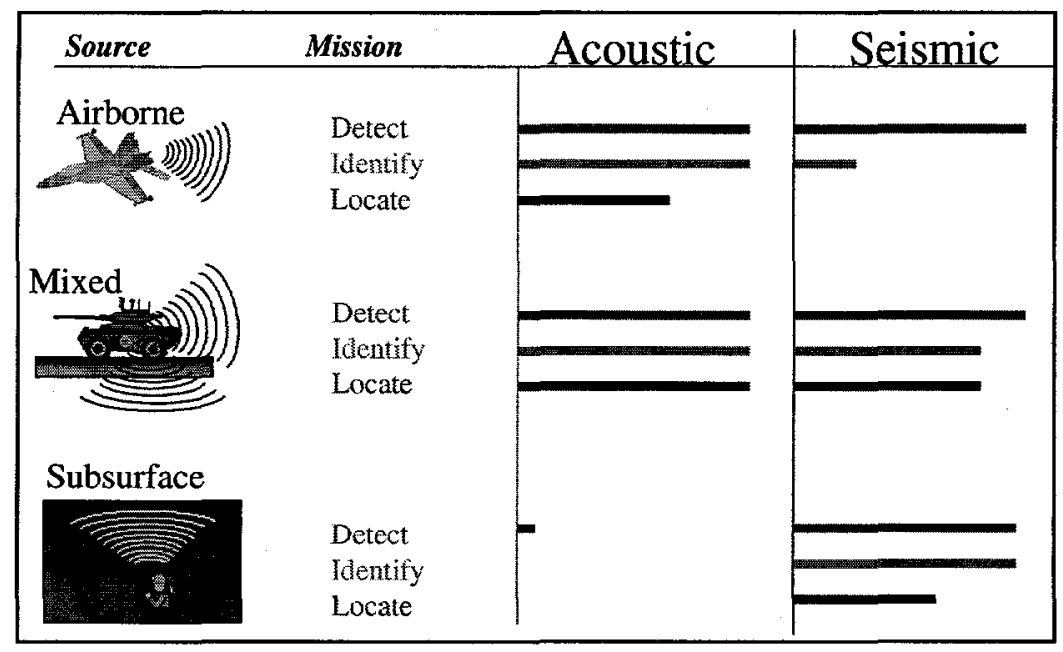

Figure 6. Methodology for selecting acoustic or seismic modalities. Some applications would benefit from a fusion of these two modalities.

\section{SUMMARY}

In this paper, we have presented the relative advantages and complementary aspects of acoustic and seismic ground sensors. A detailed description of both acoustic and seismic ground sensing methods has been provided. Acoustic and seismic phenomenology including source mechanisms, propagation paths, attenuation, and sensing have been discussed in detail. The effects of seismo-acoustic and acousto-seismic interactions as well as recommendations for minimizing seismic/acoustic cross talk have been highlighted. We have shown representative acoustic and seismic ground sensor data to illustrate the advantages and complementary aspects of the two modalities. The data illustrate that seismic transducers often respond to acoustic excitation through acousto-seismic coupling. Based on these results, we discussed the implications of this phenomenology on the detection, identification, and localization objectives of unattended ground sensors. We have concluded with a methodology for selecting the preferred modality (acoustic and/or seismic) for a particular application.

\section{ACKNOWLEDGEMENTS}

Sandia National Laboratories is a multi-program laboratory operated by Sandia Corporation, a Lockheed Martin Company, for the United States Department of Energy under Contract DE-ACO4-94AL85000. We would like to additionally acknowledge the efforts of Lawrence Livermore National Laboratory and ENSCO Inc. in support of collecting some of the data results shown in this report.

\section{REFERENCES}

1. L.E. Kinsler, et al, Fundamentals of Acoustics, $3^{\text {rd }}$ Edition, J. Wiley and Sons, New York, 1982.

2. L.G. Stotts, Steel Rattler Program Overview, DIA Internal Report, 1995.

3. N. Berg, et. al., Unattended Ground Sensor Performance Prediction and Preliminary System Requirements, joint internal report of U.S. Army Research Laboratory and Sentech, Inc. 1993.

4. G.E. Sleefe and B.P. Engler, Experimental Study of an Advanced Three-Component Borehole Seismic Receiver, $61^{\text {st }}$ Meeting of Society of Exploration Geophysicists, Houston Texas, pp. 3-33, 1991.

5. I.A. Erteza, et al, Case study: unattended ground sensor phenomenology and signal processing, Proceedings of SPIE AeroSense Conference, Vol. 3081, 1997.

6. J.P. Claassen, et al, Feasibility of monitoring continuous wave sources with seismic arrays, Proceedings of SPIE AeroSense Conference, Vol. 3713, 1999. 